\title{
The Branch Point Metabolite for Pyocyanine Biosynthesis in Pseudomonas aeruginosa
}

\author{
By D. H. CALHOUN*, MINDY CARSON AND R. A. JENSEN \\ Department of Microbiology, Baylor College of Medicine, Houston, Texas, \\ 77025 , U.S.A.
}

\section{(Received 24 February 1972; revised 5 April 1972)}

The Pseudomonas pigments are conveniently classified into the fluorescent water-soluble pigments and the chloroform-soluble phenazine derivatives. The structures of the fluorescent pigments remain largely uncharacterized, though one may be a pteridine derivative (Chakrabarty \& Roy, 1964). They are probably synthesized through a biochemical route independent of the pathway of synthesis of the phenazine compounds. The phenazine pigments are better characterized because growth media exist which reliably promote their formation.

Ingledew \& Campbell (I969) and MacDonald (I963) did precursor-product studies with ${ }^{14} \mathrm{C}$ radioisotopes to demonstrate that growing cells preferentially utilize exogenous shikimate as a precursor of pyocyanine. Shikimate was found to account for all of the carbon atoms in the ring position of the pyocyanine molecule. Similar procedures eliminated phenylalanine, tyrosine, tryptophan, and anthranilic acid as precursor molecules. The methyl group on N-5 of pyocyanine is derived from the S-methyl of methionine (Kurachi, 1969; Sheikh \& MacDonald, I964).

Since shikimate formation involves several reversible steps (the interconversion of shikimate with 5-dehydroshikimate, and of 5-dehydroshikimate with 5-dehydroquinate), 5dehydroshikimate and 5-dehydroquinate qualify a priori as candidates for the branch point metabolite. Three additional common precursors follow shikimate in the branched biosynthetic pathway leading to aromatic amino acids and vitamins. Therefore, although the labelling experiments established shikimate as a pyocyanine precursor, they did not pinpoint what particular intermediate between 5-dehydroquinate and chorismate is the branch point metabolite. Unfortunately, most of the common aromatic intermediates are unstable and/or do not penetrate the cell membrane. Thus, the labelling experiments that were suitable with shikimate, which is stable and penetrates into bacteria, could not be used to identify the branch point intermediate. The approach of using mutants blocked in the various enzyme reactions of aromatic synthesis has not been used previously. We examined the ability of such mutants to synthesize pyocyanine in the presence and the absence of shikimate.

Prototrophic mutants unable to degrade shikimate and auxotrophs (Table I) were isolated by the methods previously described (Calhoun \& Feary, 1969; Calhoun \& Jensen, 1972). Pyocyanine production was tested in the 'A' medium of King, Ward \& Raney (1954), a glycerol $-2 \%(\mathrm{w} / \mathrm{v})$ peptone medium. The $\mathrm{pH}$ of the minimal salts solution was adjusted to $7 \cdot 2$ before autoclaving. A 4 to $5 \mathrm{~h}$ culture was grown at $37^{\circ} \mathrm{C}$ with shaking in $2 \mathrm{ml}$ of trypticase soy broth (Difco) in a $15 \times 100 \mathrm{~mm}$ test tube. The culture was centrifuged, and the pellet was resuspended in $2 \mathrm{ml}$ of sterile water. Resuspended cells were spread evenly on the surface of a King agar plate in $0.2 \mathrm{ml}$ volumes and incubated at $37^{\circ} \mathrm{C}$ for 5 days. Pigment production was visible after 6 to $8 \mathrm{~h}$ for most strains, and was uniformly obvious after 24 to $36 \mathrm{~h}$.

* Present address: California College of Medicine, University of California at Irvine, Irvine, California, 92664, U.S.A. 
Table I. Pyocyanine production by mutants of Pseudomonas aeruginosa

The tryptophan auxotrophs UAB 0426, UAB 75, UAB I 35, UAB 308, ВСм I and ВСм I 3 correspond to the following blocks: anthranilate synthetase; phosphoribosyl transferase; phosphoribosyl anthranilate isomerase; indole glycerol phosphate synthetase; tryptophan synthetase A; tryptophan synthetase $\mathrm{B}$, respectively.

\begin{tabular}{|c|c|c|c|}
\hline \multirow[b]{2}{*}{ Isolate no. } & \multirow[b]{2}{*}{ Nutritional phenotype* } & \multicolumn{2}{|c|}{ Pyocyanine formation $\dagger$} \\
\hline & & $\begin{array}{l}\text { Without } \\
\text { shikimate }\end{array}$ & $\begin{array}{c}\text { With } \\
0.1 \% \text { shikimate }\end{array}$ \\
\hline Wild-type & & + & + \\
\hline UAB 446 & Leucine & + & NT \\
\hline $\mathrm{UAB} 46 \mathrm{I}$ & Methionine & + & NT \\
\hline UAB $42 \mathrm{I}$ & Histidine & + & NT \\
\hline UAB 429 & Arginine & + & + \\
\hline UAB 445 & Proline & + & NT \\
\hline UAB 4OI & Adenine & + & + \\
\hline UAB 0426 & Tryptophan/ind/ant & + & NT \\
\hline UAB 75 & Tryptophan/ind & + & NT \\
\hline UAB 135 & Tryptophan/ind & + & + \\
\hline UAB 308 & Tryptophan/ind & + & NT \\
\hline BCM I & Tryptophan/ind & + & + \\
\hline BCM I 3 & Tryptophan & + & NT \\
\hline BCM 4 & Phenylalanine/phenylpyruvate & + & + \\
\hline ВСM 20 & Shikimate degradation & + & + \\
\hline BCMN8 & Aro/shikimate/quinate & - & + \\
\hline $\mathrm{MC} 36$ & Aro/shikimate/quinate & - & + \\
\hline BCMN7 & Aro/shikimate/quinate & - & + \\
\hline BCM 40 & Aro/shikimate/quinate & - & + \\
\hline BCMN6 & Aro/shikimate/quinate & - & + \\
\hline BCM BBI & Aro/shikimate/quinate & - & + \\
\hline BCMN5 & Aro/shikimate & - & + \\
\hline BCMNI4 & Aro & - & - \\
\hline $\mathrm{MC} 42$ & Aro & & \\
\hline BCM A2 & Aro & - & - \\
\hline MC 37 & Aro & - & - \\
\hline $\mathrm{MC} 38$ & Aro & - & - \\
\hline
\end{tabular}

* Abbreviations used: aro refers to the combination of tryptophan, tryosine and phenylalanine; ind, indole; and ant, anthranilate.

† Notations: NT, not tested; + , normal pyocyanine production;,- no pyocyanine formed. The solid media used for qualitative scoring of pyocyanine production was that described by King et al. (I954).

Mutants unable to degrade shikimate as a sole source of carbon retained the ability to synthesize pyocyanine. Pigment production was also normal in a variety of amino acid auxotrophs (Table I). Pyocyanine was excreted into the agar by these mutant isolates as an intensely green to blue-green pigment. In contrast, none of the mutants requiring all three aromatic amino acids produced pyocyanine. These pyocyanine-less mutants produced only a pale yellow chromophore, even after 5 days. All of the mutants tested produced normal levels of fluorescent pigment (as judged by inspection under ultraviolet light).

Pyocyanine production was restored consistently by $0.1 \%$ (w/v) shikimate (Sigma Chemical Co.) in mutants blocked before shikimate (phenotype: alternative growth response either to shikimate or to combination of the three aromatic amino acids). On the other hand, pyocyanine synthesis was not restored by shikimate in mutants blocked after shikimate (phenotype: growth with aromatic amino acids; no growth with shikimate). Pyocyanine formation in liquid culture at $37^{\circ} \mathrm{C}$ was measured by the method of Frank \& DeMoss 
(1959). When wild-type MC36 and MC42 were grown in the presence of aromatic end products in the absence of shikimate, 65,0 and o nmol pyocyanine/ml were formed, respectively, after $8 \mathrm{~h}$ at $37^{\circ} \mathrm{C}$ (bacterial concentration, measured by $E_{525} \mathrm{~nm}$, was 0.846 to $\mathrm{I} \cdot \mathrm{I} 28 \mathrm{mg}$, dry wt, of cells $/ \mathrm{ml}$ ). In the presence of shikimate $\mathrm{r} 30, \mathrm{r} 30$ and o $\mathrm{nmol}$ of pyocyanine were formed, respectively, under the same conditions of growth.

These results allow the conclusion that shikimate or any biosynthetic precursor of shikimate can be eliminated as the branch point compound. Three enzymatic reactions convert shikimate to chorismate. Hence, the remaining branch point possibilities are shikimate-5phosphate, enolpyruvylshikimate-5-phosphate and chorismate. Although the mutant blocks have not been identified by enzyme assay, it seems likely that the five mutants that cannot convert shikimate to chorismate represent more than one mutant class. Shikimate-5phosphate can probably be eliminated since mutants lacking shikimate kinase are known to be uncommon. None of the five mutants produced pigment following starvation for aromatic end products, as might be expected if a pyocyanine precursor accumulated behind an enzyme block. The results implicate chorismate as the probable branch point metabolite for pyocyanine.

Aromatic auxotrophs that grow on shikimate cannot use chorismate to satisfy their growth requirements, indicating that significant amounts of chorismate are not transported into the cells. Accordingly, previous results (Ingledew \& Campbell, 1969) showing that chorismate did not compete with the incorporation of labelled shikimate into pyocyanine do not rule out chorismate as a precursor of pyocyanine.

Unquestionably pyocyanine represents a substantial proportion of the total output of the aromatic pathway. Hence, studies concerning the control of the regulatory enzymes in the pathway by end products must deal with the role of pyocyanine as an aromatic end product before the understanding of the physiological pattern of control can be complete.

This investigation was supported by research grant Q-422 from the Robert A. Welch Foundation, Houston, Texas, and by post-doctoral fellowship (D.H.C.) AM 365I 7 from the U.S. Public Health Service.

\section{REFERENCES}

Calhoun, D. H. \& Feary, T. W. (1969). Transductional analysis of Pseudomonas aeruginosa methionine-less auxotrophs. Journal of Bacteriology 97, $210-216$.

Calhoun, D. H. \& Jensen, R. A. (1972). Significance of altered carbon flow in aromatic amino acid synthesis: an approach to the isolation of regulatory mutants in Pseudomonas aeruginosa. Journal of Bacteriology rog, $365-372$.

Chakrabarty, A. M. \& Roy, S. C. ( 1964). Characterization of a pigment from a pseudomonad. Biochemical Journal 93, I 44-148.

FRANK, L. H. \& DEMoss, R. D. (1959). On the biosynthesis of pyocyanine. Journal of Bacteriology 77, 776782 .

Ingledew, W. M. \& CAMPbell, J. J. R. (I969). Evaluation of shikimic acid as a precursor of pyocyanine. Canadian Journal of Microbiology $\mathbf{1 5}, 535-54 \mathrm{I}$.

King, E. O., WARD, M. K. \& RaneY, D. E. (I954). Two simple media for the demonstration of pyocyanine and fluorescein. Journal of Laboratory Clinical Medicine 44, 30I-307.

Kurachi, M. (1959). Studies on the biosynthesis of pyocyanine. IV. On the effect of methionine and other promoting factors in peptone. Bulletin of Institute of Chemical Research, Kyoto University 37, 48-58.

MaCDonald, J. C. (I963). Biosynthesis of pyocyanine. Canadian Journal of Microbiology 9, 809-819.

Sheikh, N. M. \& MacDonald, J. C. (1964). Biogenesis of the N-methyl group of pyocyanine. Canadian Journal of Microbiology 1o, 86I-866. 